\title{
Ontologias e tecnologia da informação e comunicação: sistemas especialistas, web semântica e gestão integrada de compras governamentais eletrônicas
}

DOI: $10.3395 /$ reciis.v1i1.47pt

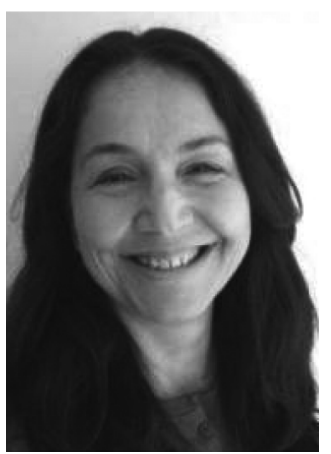

Laura Cristina Simões Viana Vice-Presidência de Pesquisa e Desenvolvimento Tecnológico da Fundação Oswaldo Cruz Rio de Janeiro, Brasil

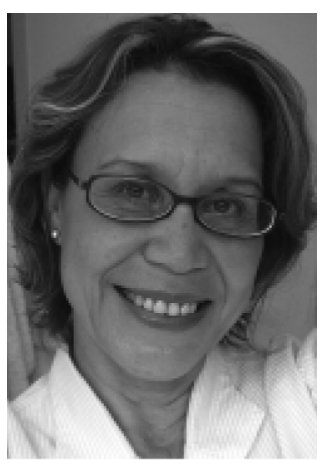

Olga Fernanda Nabuco

Centro de Pesquisas Renato Archer - CENPRA Campinas, Brasil / Laboratoire D'Analyse Et D'Architecture Des Systemes - Centre National de la Recherche Scientifique LAAS/CNRS (CNRS), Toulouse, França

\section{Carlos José Saldanha Machado}

Fundação Oswaldo Cruz, Rio de Janeiro, Brasil

csaldanha@cict.fiocruz.br

\section{Resumo}

A consolidação da tecnologia da informação e comunicação como um paradigma técnico-econômico sinaliza transformações no modo de se fazer ciência. Tanto que as pesquisas de ponta exigem expressivas capacidades de processamento e de rede para a manipulação de dados originados de diversas disciplinas e dispersos geograficamente, inaugurando a "e-ciência", a exemplo da bioinformática. Nesse contexto, o presente trabalho apresenta os Sistemas Especialistas e a Web Semântica, cuja construção depende de tecnologias de representação do conhecimento, a exemplo das ontologias. Estas, ao explicitarem uma conceituação comum, podem viabilizar a integração de conjuntos de dados diversos e heterogêneos, facilitando a interoperabilidade dos sistemas de informação. Esta integração de dados é uma etapa crítica do desenvolvimento de quase todos os sistemas computacionais, sejam estes utilizados em atividades de pesquisa, como na nova organização do trabalho colaborativo, a "e-ciência", como em atividades governamentais e também comerciais. Conclui-se afirmando que a construção de ontologias é uma alternativa tecnológica para a interoperabilidade dos sistemas de informação, podendo favorecer a nova organização do trabalho colaborativo em prol de uma gestão integrada de compras governamentais eletrônicas, particularmente no Setor Saúde.

\section{Palavras-chave}

Ontologias, sistemas especialistas, web semântica, tecnologia da informação, compras governamentais 


\section{Introdução}

Quando a biotecnologia moderna, caracterizada pela possibilidade de manipulação direta do código genético, se mostrou um paradigma tecnológico ${ }^{1}$ na década de 1980, a microeletrônica já se configurava como um novo paradigma técnico e econômico ${ }^{2}$. Inicialmente restrita aos modernos laboratórios de pesquisas, especulava-se sobre o potencial revolucionário da nova biotecnologia e sobre o mercado que essa tecnologia representaria para a microeletrônica (PEREZ, 1986). Atualmente, a Tecnologia de Informação e Comunicação - TIC - de base microeletrônica é considerada uma tecnologia genérica (FREEMAN, 2003), pois, além de ter demonstrado inúmeras vantagens técnicas e econômicas, vencendo a barreira do mercado, avança por toda a sociedade e determina mudanças também nas dimensões organizacional e institucional. Por outro lado, as pesquisas mais recentes no campo da biotecnologia invocam a "e-ciência" (e-science), inaugurando a bioinformática e confirmando a vocação da biotecnologia como mercado para a $\mathrm{TIC}^{3}$.

O termo e-science 4 ou "e-ciência" foi cunhado por John Taylor, do Escritório de Ciência e Tecnologia do Reino Unido - OST (HEY et al., 2002), para traduzir uma nova organização do trabalho científico, caracterizada pela colaboração global em áreas-chave da ciência e seu equivalente em infra-estrutura necessária à operacionalização da “e-ciência”. Segundo HEY et al. (2002), a enorme quantidade de bytes gerados, diariamente, pela comunidade científica e tecnológica, exige o acesso eficiente aos dados armazenados, que se encontram dispersos geograficamente, bem como expressiva capacidade computacional e de rede para a gestão e a análise desses dados em prazos e custos factíveis.

Para atender a esta demanda, surgiu o conceito de Grid computing (HEY et al., 2002), ou computação distribuída, que é um modelo computacional de processamento distribuído em uma infra-estrutura que executa, em simultâneo, a mesma tarefa em diversos processadores. A idéia subjacente ao termo Grid computing é análoga à de rede de energia elétrica, onde geradores de energia elétrica são distribuídos e os usuários têm acesso à energia elétrica sem se preocupar com a fonte de energia e sua localização. Ou seja, este modelo envolve uma série de computadores de menor porte, conectados entre si via redes locais e Internet, os quais conformam uma arquitetura virtual de computadores que, quando ociosos, realizam atividades que exigem alto desempenho.

Esta nova abordagem da computação em rede é também conhecida como metacomputação (metacomputing), computação expansível (scalable computing), computação global (global computing), computação via Internet (Internet computing) e computação ponto a ponto (peer-to-peer computing) (BAKER et al., 2002).

O espaço da "e-ciência" não se limita aos megaprojetos ou à ciência fundamental. Há indicações atuais de que a "e-ciência" está modificando a maneira como as pessoas colaboram, ou extraem conhecimentos de enormes quantidades de dados, ou organizam projetos de engenharia e de negócios (REDFEARN, s.d.). A “e-ciência” se constitui numa inovação na organização do trabalho científico, formatando uma nova infra-estrutura para a produção de conhecimentos científicos (HINE, 2006). Entretanto, o desenvolvimento da "e-ciência" e seus desdobramentos necessitam da integração de conjuntos de dados diversos e heterogêneos organizados em diferentes disciplinas. $\mathrm{O}$ compartilhamento de ontologias definidas, para fins de simplificação como conceituações comuns e seus relacionamentos, pode auxiliar esta integração.

O presente trabalho apresenta os Sistemas Especialistas - SEs - e Web Semântica - WS. Estas tecnologias são exemplos de TIC, cujo desenvolvimento depende da construção e da reutilização de conceituações comuns. Após esta introdução, o trabalho segue com uma breve caracterização e retrospectiva da evolução dos trabalhos em Inteligência Artificial - IA, área na qual as possibilidades de representação do conhecimento são uma questão importante para o sucesso da tarefa em dotar uma máquina com capacidade de inferência.

A segunda seção apresenta uma visão geral dos Sistemas Especialistas - SEs - e suas respectivas aplicações para mostrar, em seguida, uma nova versão da Web, a Web Semântica - WS - e como esta poderá ser praticada. Na penúltima seção, o trabalho caracteriza o termo ontologia no contexto da TIC e explora algumas de suas aplicações mais recentes. Finalmente, o trabalho sugere um exercício de elaboração de uma ontologia de produtos e serviços que possa ser utilizada como ferramenta de auxílio às atividades de compras governamentais.

\section{Inteligência artificial e sistemas espe- cialistas}

A partir do trabalho fundamental de TURING, MCCARTHY e MINSKY foi cunhado o termo Inteligência Artificial - IA, e lançada a pedra fundamental da utilização de máquinas que aprendem e inferem, facilitando o trabalho e a vida do homem (MCCARTHY et al., 1969; MCCARTHY, 2004; MACKWORTH, 2007). A linha de pesquisa em IA simbólica ${ }^{5}$, que segue a tradição lógica inaugurada por McCarthy e Hayes, entende que um programa de computador "inteligente" deve ter uma representação geral do mundo a partir da qual suas entradas podem ser interpretadas; do ponto de vista prático, o problema da IA é como modelar a "inteligência" (BITTENCOURT, 2005). A evolução da IA simbólica pode ser dividida em três fases (BITTENCOURT, 2005): clássica, romântica e moderna. $\mathrm{Na}$ fase clássica, que se prolongou até a década de 1970, o objetivo da IA simbólica era simular a inteligência humana, utilizando-se de métodos solucionadores gerais de problemas e lógica. Nesta fase, a principal limitação da IA foi subestimar a complexidade computacional dos problemas. A fase romântica, entre os anos de 1970 e de 1980, tinha por objetivo simular a inteligência humana em situações predeterminadas. Os métodos adotados nessa fase eram os formalismos de representação de conhecimento adaptados ao tipo de problema e os mecanismos de enfoque procedural $^{6}$, com o objetivo de atingir maior eficiência computacional. A fase romântica não tinha a noção da 
quantidade de conhecimento necessário para lidar com problemas simples de senso comum.

O surgimento dos Sistemas Especialistas - SEs - marcou a passagem da IA para a fase moderna entre as décadas de 1980 e 1990. Nesta fase, o objetivo da IA simbólica era forjar o comportamento de um especialista, em um domínio específico, na resolução de problemas. Assim, a partir de 1980 vários SEs foram desenvolvidos, mas, após este sucesso inicial, seguiu-se um desencanto, reforçando o entendimento de que a inteligência em um Sistema Especialista - SE - encontra-se, essencialmente, na forma como o conhecimento sobre o domínio é representado e que a etapa de aquisição do conhecimento é a parte mais difícil do desenvolvimento de um sistema (BITTENCOURT, 2005; FURNIVAL, 1995).

Os parágrafos a seguir situam esta questão da representação do conhecimento no contexto da IA simbólica, caracteriza os SEs e enumera alguns exemplos e suas respectivas áreas de aplicação.

Os métodos (ou formalismos) clássicos de representação do conhecimento, cuja base é a Lógica (ou Lógica de primeira e segunda ordem), são os sistemas de produção, as redes semânticas e os sistemas de representação de quadros (BITTENCOURT, 1998). Os sistemas de produção incluem os sistemas baseados em regras de produção, ou seja, pares de expressões consistindo em uma condição "se" e uma ação "então". As redes semânticas, utilizadas em sistemas para a compreensão da linguagem natural, são um conjunto de nodos conectados por conjuntos de arcos, onde os primeiros representam objetos e os últimos, relações binárias entre os objetos. Os quadros são nodos com estrutura interna; este último método é considerado a base das idéias que resultaram nas linguagens de programação orientadas ao objeto. Os SEs utilizam regras de produção como método de representação do conhecimento.

Considerando que a capacidade cognitiva pode ser dividida em duas partes, uma base de conhecimento declarativa e um motor de inferência (BITTENCOURT, 1998), os SEs igualmente possuem dois componentes principais, que são a sua base de conhecimento e o motor (ou máquina) de inferência (ou de raciocínio). A base de conhecimento reúne dados factuais ou regras e o motor de inferência aplica as regras para inferir novos conhecimentos.

Os sistemas DENDRAL e MYCIN são exemplos de SEs clássicos (LUGER, 2005). O sistema DENDRAL é emblemático dos tempos pioneiros dos SEs voltados para a resolução de problemas em domínios específicos. Desenvolvido em Stanford no fim dos anos de 1960, como um conjunto de programas, o objetivo do sistema DENDRAL era inferir a estrutura de moléculas orgânicas com base em fórmulas químicas e em dados de espectrometria de massa sobre as ligações químicas presentes nas moléculas (LINDSAY et al., 1993).

O sistema DENDRAL adota um método de busca heurística e conhecimento químico especializado na realização da busca, limitando a procura a situações promissoras (WALKER, 1987). Embora o DENDRAL seja conhecido na comunidade da química computacional, a sua utilização na prática da química foi limitada e, enquanto um pacote integrado de software, não é mais executado (LINDSAY et al., 1993). A experiência adquirida com o DENDRAL mostrou-se relevante para a IA e para o projeto e a implementação de outros sistemas especialistas (LINDSAY et al., 1993).

O sistema MYCIN, também desenvolvido em Stanford em meados dos anos de 1970, inaugurou a metodologia contemporânea dos SEs ao solucionar os problemas de raciocínio com informação incerta e incompleta (LUGER, 2005). Este sistema fornece explicações lógicas sobre o seu raciocínio, usa uma estrutura de controle adequada ao domínio específico do problema e identifica critérios confiáveis para avaliar seu próprio desempenho (SHORTLIFFE, 1984; 1975). O sistema MYCIN utiliza conhecimento médico especializado no tratamento de pacientes acometidos de meningite e de bacteremia (SHORTLIFFE, 1984). Junto com o MYCIN, os sistemas PIP, INTERNIST-1 e CASNET, brevemente descritos a seguir, são considerados marcos inaugurais das pesquisas em IA aplicadas em Medicina (SHORTLIFFE, 1986):

- Sistema PIP (Present Illness Program): reúne dados para gerar hipóteses sobre processos de doenças em pacientes com doenças renais.

- Sistema INTERNIST-1: utilizado no diagnóstico de problemas complexos em medicina interna (SHORTLIFFE, 1986).

- Sistema CASNET: é um sistema de assessoria em oftalmologia utilizado em avaliações da doença e na administração de pacientes com glaucoma.

Atualmente, as aplicações de IA na prática da Medicina $^{7}$ incluem a prescrição de medicamentos, os laboratórios clínicos, os contextos educacionais, o acompanhamento clínico e as áreas que exigem a manipulação de muitos dados, como unidades de terapia intensiva (COIERA, 2003).

Os sistemas PROSPECTOR, DIPMETER ADVISOR e XCON, adiante enumerados, também são considerados SEs clássicos (LUGER, 2005):

- Sistema PROSPECTOR: projetado para localizar depósitos de minerais, como ouro e molibdênio (WALKER, 1986).

- Sistema DIPMETER ADVISOR: utilizado para interpretação de resultados de perfuração de poços de petróleo.

- Sistema XCON: utilizado na configuração de computadores VAX (Digital Equipment Corporation).

A utilização dos sistemas especialistas pode contribuir para melhorias na produtividade em atividades comerciais, científicas, tecnológicas e militares. O mercado oferece, atualmente, inúmeros pacotes comerciais de sistemas especialistas com interfaces amigáveis, os quais possuem várias aplicações (ENGELMORE, 1993):

- diagnóstico e identificação de problemas em dispositivos e sistemas em áreas diversas, como sistemas médicos e de engenharia; 
- planejamento e elaboração de cronogramas, como programação de vôos, de escala de pessoal e de terminais de embarque e desembarque para companhias aéreas, programação de job-shop de manufatura e planejamento de processo de manufatura;

- configuração de objetos manufaturados através de montagens parciais, como a construção de casas modulares e outras atividades que envolvem projeto de engenharia e de manufatura complexos;

- tomada de decisão no setor financeiro, como programas de assessoria utilizados em suporte a análise de crédito, análise de risco e determinação do prêmio em seguros, e comércio exterior;

- editoração de conhecimento, como assessoria em ortografia e gramática e assessoria tributária, em especial imposto de renda pessoa física;

- controle e monitoramento de processos, como os sistemas utilizados em usinas siderúrgicas e em refinarias de petróleo, os quais analisam, em tempo real, dados originados dos equipamentos e outros dispositivos, com o objetivo de detectar anomalias, prever tendências e controlar a correção de falhas e a otimização;

- planejamento e manufatura, como os sistemas de auxílio a projetos de produtos e de processos tanto no plano conceitual como na configuração de chão de fábrica de processos de manufatura;

- serviços de informação e bibliotecas, como os sistemas utilizados em indexação, formulação automática de resumos, trabalhos de referência, catalogação, recuperação de informações on-line, desenvolvimento de coleções, detecção de registros duplicados (FURNIVAL, 1995; MENDES, 1997).

Alguns exemplos de pesquisas sobre sistemas especialistas realizadas no Brasil incluem aplicações em Medicina (RAZZOUK et al., 2006); em projeto de produto (MAZIERO, 2000); no apoio à tomada de decisão em ambiente industrial (HENNEMANN, 2006); no uso e manejo da terra (GIBOSHI et al., 2006); no gerenciamento da informação (MENDES, 1997); no controle de processo industrial (SELLITO, 2002); no controle sanitário de sementes (ALVES et al., 2006) e na avaliação de terras para o cultivo de grãos (CHAGAS et al., 2006). Grande parte dos produtos comerciais disponíveis no mercado brasileiro que utilizam IA combinam recursos de sistemas especialistas (lógico) e de redes neurais (intuitivo) com aplicações, principalmente, nas áreas financeira, de telecomunicações e de serviços de utilidade púbica, como energia (ABES, s.d.).

Antes de passarmos para a próxima seção, sobre a Web Semântica, convém relembrar que a etapa mais crítica da construção de um SE é aquela de aquisição do conhecimento, que consiste na acumulação, transferência e transformação do conhecimento para a máquina, formando a base de conhecimento do SE (CIN/UFPE, s.d.). As principais fases da aquisição do conhecimento na construção de um SE são a identificação das características do problema, cuja resolução é objeto do SE, o desenvolvimento de uma ontologia (conceituações comuns) no domínio do problema e a identificação das inferências sobre os conceitos formadores da ontologia. Mais recentemente, a aquisição do conhecimento para os SEs passou a ser guiada por modelos, os quais incluem a reutilização de ontologias e estruturas de inferência (CIN/UFPE, s.d.).

\section{Web semântica}

Embora a Web seja cada vez mais parecida com a TV, é inegável que a Web permanece interativa e rica em informações, possibilitando a conexão de um sem-número de dispositivos, como a casa inteligente e as consultas à conta bancária através do celular. Entretanto, existe um caminho a ser percorrido para que dispositivos diferentes e pessoas possam conectar-se, pois a Web não é mais limitada a uma interface de computador.

Dado que a Web se desenvolveu como um repositório de documentos para a manipulação por pessoas e não por máquinas, a exploração de todo o potencial da Web deve privilegiar uma linguagem que seja compreensível para as pessoas e para as máquinas. Uma solução possível repousaria em uma teoria semântica que interpretasse símbolos, transformando dados em informações e fornecendo explicações sobre os significados; a conexão lógica dos termos estabeleceria a interoperabilidade entre os sistemas (BERNERS-LEE et al. 2001). A Web Semântica - WS - é uma nova versão ou uma versão ampliada da Web atual, onde a informação é acompanhada por um significado bem definido, auxiliando o trabalho cooperativo entre pessoas e computadores.

Segundo BERNERS-LEE et al. (2001), o funcionamento da WS depende dos computadores terem acesso a coleções estruturadas de informações e conjuntos de regras de inferência que possam ser utilizadas na condução da automação da inferência do raciocínio automatizado. Conforme comentado anteriormente na seção II, a inteligência em um sistema encontra-se na representação do conhecimento. O desafio da WS, portanto, é desenvolver uma linguagem que expresse dados e regras de raciocínio sobre os dados, permitindo que as regras de qualquer sistema de representação de conhecimento sejam exportadas para a Web. O que é, então, necessário para descrever uma informação de modo que esta seja igualmente compreensível para pessoas e máquinas? O modelo em camadas de padrões e tecnologias Web, mostrado na Figura 1 e descrita nos parágrafos seguintes, sugere uma arquitetura para a WS.

As recomendações da $\mathrm{W}^{3} \mathrm{C}^{8}$ indicam que os recursos $^{9}$ apontados pelos endereços não devem possuir significados dúbios, mas sim relacionar dados com objetos do mundo real. Os endereços eletrônicos de projetos encontram-se na camada Uniform Resource Identifier - URI, a qual fornece de modo inequívoco os meios para a identificação de recursos na Web. Uma URI é um nome (string) curto que identifica recursos na Web, como documentos, imagens, endereços de arquivos "descarregáveis", serviços, endereços eletrônicos, entre outros. A URI é uma forma genérica de Uniform Resource Location - URL, o endereço tradicional da Web, como por exemplo, www.w3c.org. 
Figura 1 - Web semântica. Modelo em camada

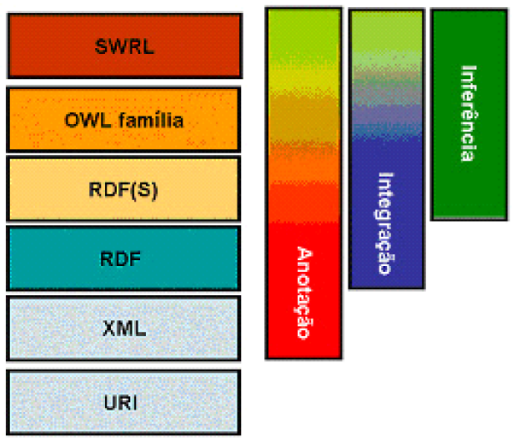

Fonte: adaptado de Globe (2007)

As camadas eXtensible Markup Language - XML - e Resource Description Framework - RDF - representam os formatos de dados e informações usuais para a Web. A camada XML, junto com definições de tipos e esquemas, garante a integração das definições de WS com outros padrões baseados em XML. O RDF é modelo universal de formato para dados na Web, permitindo que dados estruturados e semi-estruturados sejam misturados, exportados e compartilhados por diferentes aplicações. O Resource Description Framework descreve vários tipos de recursos, enquanto os esquemas XML, por exemplo, descrevem apenas documentos, possibilita a interoperabilidade entre aplicações e permite que o significado dos termos e dos conceitos seja prontamente processado pelos computadores (BERNERS-LEE et al., 2001); pode utilizar XML em sua sintaxe e URI para descrever entidades, conceitos, propriedades e relações.

A linguagem RDFSchema contém esquemas que facilitam a recuperação de informações no formato adequado para uma determinada aplicação, tornando possível aos programas de computador "entender" as informações. A RDFSchema define classes e propriedades específicas às aplicações, sendo que as classes podem ser coleções de recursos. A camada Web Ontology Language - OWL - utiliza Lógica de Descrição (Lógica de primeira ordem) e pretende estender o significado das aplicações que utilizam a Web para programas que interpretam automaticamente essas informações. O objetivo é inserir a interoperabilidade de máquinas, programas automáticos, agentes ${ }^{10}$ etc. com sistemas Web.

A linguagem OWL permite que se faça inferência sobre o conteúdo que ela representa. Semantic Web Rule Language - SWRL - é uma linguagem para escrever regras em lógica de primeira ordem. Utilizada em conjunto com OWL, forma uma dupla potente de linguagem de representação do conhecimento para Web. SWRL e OWL juntas permitem guardar informação categorizada e recuperá-la. Além disso, também permitem que um motor de inferência possa usar essa base para acrescentar novos conhecimentos à própria base.

Anotações são feitas sobre a base de conhecimento principal, acrescentando-se um link para uma informação que explicite mais ainda esta informação. A camada Anotação acrescenta informação à própria informação, permitindo distingui-la de outras informações, caracterizando-a melhor. A camada Integração porta interoperabilidade para as aplicações, integrando fontes de informações; em termos semânticos, a integração traz à tona o significado exclusivo da informação, que extrapola aquele de uma simples palavra-chave ou de um thesaurus, aproximando-se de um catálogo do tipo "páginas amarelas". A cada camada aumenta o reconhecimento do valor da informação. A inferência é possível quando a linguagem de descrição é rica o suficiente para descrever axiomas e frases lógicas, permitindo que um programa do tipo sistema especialista ou uma máquina de regras interprete a informação, acrescentando-lhe nova informação. Esta nova informação, embora ainda não estivesse escrita, obedece às regras existentes. A camada Inferência raciocina com base na informação existente e cria novas instâncias da informação. Esta camada não cria novas regras, a exemplo das conclusões construídas a partir de propriedades de transitividade: se Flávio Simões é filho do Luiz Simões e cada pessoa pode ter um só pai, e João Barbosa é pai do Flávio Simões, então, conclui a máquina, Luiz Simões e João Barbosa são a mesma pessoa. Em resumo, o desenvolvimento da Web Semântica concentra-se na definição de camadas de linguagens utilizadas no suporte à representação e utilização de metadados; as linguagens constituem o instrumental básico utilizado para acrescentar significado à informação necessária para a Web Semântica (GLOBE, 2007).

As aplicações da Web Semântica são inúmeras, e as indicações a seguir constituem uma amostra: biblioteca de metadados (para integração em um único domínio); integração de dados em segurança pública; integração de dados de P\&D; ferramentas de busca; conexão de conhecimentos de diferentes disciplinas, como genômica, proteômica, ensaios clínicos, regulamentação e outras; governo eletrônico e energia (HERMAN, 2007). Em termos de página na Internet, o Mind Lab ${ }^{11}$, do Institute for Advanced Computer Studies, da Universidade de Maryland, combina tecnologias Web (HTML, XHTML, XML, PHP, CSS etc.) com linguagens WS (RDF, RDFS, DAML+OIL, OWL) e outras ferramentas. O projeto FOAF - Friend of a Friend ${ }^{12}$, cuja proposta é criar páginas da Web pessoais que também possam ser lidas por máquinas, igualmente incorpora tecnologias WS. Já o centro Biotechnological Centre - BIOTEC da Technische Universität Dresden desenvolveu uma máquina de busca em ciências da vida, e em particular em biologia molecular, GoPubMed ${ }^{13}$, baseada no serviço PubMed ${ }^{14}$. Os resultados das buscas são classificados de acordo com GeneOntology ${ }^{15}$, que é um vocabulário controlado para descrição do gene e dos atributos de seus produtos em qualquer organismo. Ao mesmo tempo, alguns produtos comerciais já utilizam tecnologias $\mathrm{WS}^{16}$ desenvolvidas pelo Consórcio W3C.

Estas iniciativas ainda guardam alguma distância de um modelo de Web de dados e informações, ou seja, da Web Semântica. Este distanciamento ocorre porque ainda não existe mediação baseada em agentes, em larga escala, a qual depende da consolidação de padrões e tecnologias Web que descrevam significados 
(SHADBOLT et al., 2006). Por outro lado, a utilização crescente de ontologias na "e-ciência" para a integração de conjuntos de dados distintos e heterogêneos, oriundos de diversas áreas, pode acelerar o desenvolvimento da WS (SHADBOLT et al., 2006). Outros especialistas, como LÉVY (2006), argumentam, entretanto, que a WS não resolve a questão da interoperabilidade semântica ${ }^{17}$ porque a notação de conceitos em linguagem natural é arbitrária e também porque as inúmeras ontologias são incompatíveis.

\section{O que significa ontologia}

Duas questões inter-relacionadas estão presentes em muitas das discussões sobre Inteligência Artificial: a representação do conhecimento e a interoperabilidade. A primeira, já comentada na seção II, trata da representação do mundo (ou parte dele), de um modo tal que as máquinas possam processá-lo. Esta questão constituiuse em uma área específica de estudos, a engenharia do conhecimento, formada a partir do desenvolvimento dos Sistemas Especialistas (WELTY, 2003). A outra questão, mais recente, é a interoperabilidade entre os sistemas de informação, a qual ainda tem um longo caminho a percorrer para viabilizar a comunicação entre os sistemas e também entre estes e seus usuários. Em ambas as questões, as ontologias desempenham um papel fundamental.

Existem muitas construções denominadas ontologias que se diferenciam no propósito da modelagem, da representação utilizada para a modelagem e do ponto de vista filosófico (BODENRIDER et al., 2006). O termo ontologia, originário da Filosofia, foi incorporado pela Ciência da Computação no começo dos anos de 1980, quando John McCarthy o utilizou pela primeira vez (WELTY, 2003). Desde então, surgiram muitas definições para ontologia, sem que se tenha notícia, até o momento, de um consenso sobre o termo na Ciência da Computação.

Segundo WELTY (2003), a ontologia na Ciência da Computação trabalha o significado e a existência de objetos e conceitos; para o autor, a ontologia define os objetos e os conceitos que existem no domínio de um sistema, como estes se relacionam e qual é o significado mais claro possível destes objetos e conceitos. Para GRUBER (1983), importa a finalidade da ontologia e, no contexto do compartilhamento e da reutilização do conhecimento, a ontologia pode ser definida como uma especificação explícita de uma conceituação. Ou seja, a ontologia é uma descrição dos conceitos e dos relacionamentos que podem existir entre eles. Nesse contexto, a especificação da ontologia cria compromissos ontológicos, os quais significam um acordo sobre a adoção de um vocabulário consistente, embora incompleto, com relação à teoria especificada por uma ontologia. Segundo GRUBER (1983), a conceituação é a base de um corpo de conhecimento formalmente representado. Para STUDER et al. (1998), a conceituação refere-se a um modelo abstrato de um fenômeno, identificando os conceitos relevantes relativos a esse fenômeno; a especificação explícita indica que os tipos de conceitos utilizados e as restrições referentes à utilização dos respectivos conceitos são explicitamente definidos.

Segundo SHADBOLT et al. (2006), nos últimos cinco anos, os projetos em Web Semântica apontam para a necessidade de desenvolvimento, gestão e validação de ontologias, independentemente do domínio. A reutilização de ontologias e dados deverá conduzir os usuários à reutilização e à descoberta de informações, movimentos estes que podem apresentar grandes desafios para as pesquisas em Web Semântica. Por exemplo, poderá ser necessário procurar auxilio em áreas diversas, como análise de redes sociais e epidemiologia, para entender como a informação e os conceitos se espalham na Web e como garantir a origem e a confiabilidade da informação e dos conceitos (SHADBOLT, 2006). Os desenvolvimentos, as metodologias, os desafios e as técnicas em discussão sobre a Web Semântica contribuirão para a construção de uma nova Web e, segundo SHADBOLT (2006) e BERNERSLEE et al. (2006), para uma nova disciplina: a Ciência Web. A Ciência Web trataria de desenvolver, implementar e entender sistemas distribuídos de informação, sistemas de pessoas e sistemas de máquinas operando em escala global (SHADBOLT, 2006).

\section{Aplicações práticas de ontologias}

Tal como comentado na introdução deste trabalho, os projetos de pesquisa, desenvolvimento e inovação na biotecnologia moderna são multidisciplinares e demandam a integração de conjuntos de dados diversos e heterogêneos, tornando-se um dos principais mercados para a Tecnologia da Informação e Comunicação e inaugurando a bioinformática. Esta integração pode ser favorecida com a adoção de ontologias comuns. Tanto que, nos últimos anos, o desenvolvimento de ontologias de uso específico em determinadas disciplinas tornou-se uma realidade, permitindo que especialistas de um domínio compartilhem e anotem informações em suas respectivas áreas de expertise (NOY et al., 2001). As ontologias estão sendo adotadas não só por comunidades científicas, mas também em atividades comerciais ${ }^{18}$ e governamentais, como uma forma de compartilhar, reutilizar e processar o conhecimento sobre um domínio.

Por exemplo, na área da medicina ${ }^{19}$, foi desenvolvido o Systematized Nomenclature of Medicine - SNOMED ${ }^{20}$, que é um vocabulário estruturado e padronizado, e a rede semântica Unified Medical Language System - UMLS ${ }^{21}$. $\mathrm{Na}$ área biomédica, a página Open Biomedical Ontologies - $\mathrm{OBO}^{22}$ - reúne vocabulários estruturados para uso compartilhado entre diferentes domínios das áreas biológica e médica. A OBO contém ontologias ${ }^{23}$ genéricas aplicadas a todos os organismos e ontologias de escopo mais restrito, para serem utilizadas em grupos taxonômicos específico. Já a página do projeto The Gene Ontology - $\mathrm{GO}^{24}$ - apresenta um vocabulário controlado para descrever genes e atributos dos produtos dos genes em quaisquer organismos. Outras disciplinas igualmente procuram integrar dados e informações, a exemplo do Plant Ontology ${ }^{\mathrm{TM}}$ Consortium $^{25}$, que desenvolve, acompanha e compartilha ontologias sobre estruturas de plantas e seus estágios de crescimento e desenvolvimento, e do 
projeto Marine Metadata Interoperability ${ }^{26}$, que promove o intercâmbio, a integração e o uso de dados em ciências do mar. A construção da Linguagem de Marcação da Plataforma Lattes ${ }^{27}$ - LMPL - é uma iniciativa brasileira voltada para a integração de sistemas de informações em ciência e tecnologia através da construção de uma ontologia comum neste domínio (PACHECO et al., 2001).

Na área governamental, a Diretiva 2003/98/EC, da Comissão Européia, estabeleceu condições gerais para a reutilização de documentos produzidos pelo setor público dos países Membros e para o acesso a estes documentos. Esta Diretiva visa facilitar a ampla circulação de informações sobre produtos e serviços contidos nos documentos, inclusive no ambiente empresarial, promovendo, desta forma, a competição (EU, 2003).

No Reino Unido, o estabelecimento de políticas sobre padrões, acesso e fomento à reciclagem de informações do setor público é de responsabilidade do Office of Public Sector Information ${ }^{28}$ - OPSI. Também no Reino Unido, a página GovTalk ${ }^{29}$ é um fórum de trabalho conjunto para o desenvolvimento de políticas e padrões para o governo eletrônico; a página Electronic Service Delivery - ESD ${ }^{30}$ - disponibiliza listas controladas e padrões de dados associados, utilizados em todas as esferas do setor público; o vocabulário The Integrated Public Sector Vocabulary - IPSV ${ }^{31}$ - apresenta um esquema de codificação, utilizado na marcação de informação, visando garantir o fluxo transparente das informações entre os órgãos do setor público e oferecer aos cidadãos e empresários melhor acesso aos serviços públicos.

Pode-se também encontrar ontologias de uso geral, como a taxionomia The United Nations Standard Products and Services Code ${ }^{\circledR}$ - UNSPSC ${ }^{32}$, que reúne uma classificação aberta para produtos e serviços em geral. O código UNSPSC é uma ferramenta de negócios que tem padrão aberto e, portanto, suas especificações estão disponíveis. O objetivo deste código é auxiliar a atividade de compras de empresas e instituições, consolidando valores e relacionando-os a produtos e fornecedores. $\mathrm{O}$ código UNSPSC é compatível com outros sistemas e permite análises agregadas e desagregadas em diversas etapas da atividade de compras, inclusive compras eletrônicas. Os segmentos incluídos no UNSPSC são: matérias-primas, equipamentos industriais, produtos finais, serviços e componentes.

\section{Exercício de elaboração de uma onto- logia para gestão de compras gover- namentais eletrônicas}

Antes de terminar este trabalho, sugerimos um exercício de elaboração de uma ontologia que possa ser utilizada por organismos da Administração Pública Brasileira como ferramenta de auxílio às atividades de compras governamentais eletrônicas, as quais representam 57\% do total contratado pelo governo federal (BRASIL, 2007). Estima-se que R\$ 2 bilhões, ou 22\% desse total, sejam referentes ao Setor Saúde ${ }^{33}$.

De acordo com a Constituição Federal, art. 37, inciso XXI, as obras, serviços, compras e alienações no âmbito da Administração Pública Brasileira devem ser contratadas mediante processo de licitação pública que assegure igualdade de condições a todos os concorrentes. $\mathrm{O}$ arcabouço regulamentar básico da atividade de compras governamentais compreende:

- Lei no 8.666, de 21 de junho de 1993, que regulamentou o artigo 37, inciso XXI, da Constituição Federal, e instituiu normas para licitações e contratos da Administração Pública (BRASIL, 1993) ${ }^{34}$.

- Decreto no 3.555, de 08 de agosto de 2000, que regulamentou o Pregão para a Administração Federal (BRASIL, 2000).

- Lei $\mathrm{n}^{\mathrm{o}}$ 10.520, de 17 de junho de 2002, que instituiu, no âmbito da União, Estados, Distrito Federal e Municípios, também nos termos do artigo 37, inciso XXI, da Constituição Federal, a modalidade de licitação denominada Pregão, para aquisição de bens e serviços comuns (BRASIL, 2002); e

- Decreto no 5.450, de 31 de maio de 2005, tornou o Pregão obrigatório nas contratações públicas no Governo Federal, dando preferência o eletrônico (BRASIL, 2005).

A modalidade de licitação denominada Pregão é destinada à aquisição de bens e serviços comuns, assim considerados aqueles cujos padrões de desempenho e qualidade possam ser objetivamente definidos por meio de especificações amplamente conhecidas no mercado. Incluem-se, neste grupo, cerca de 50 mil tipos de produtos e 2,5 mil modalidades de serviços (BRASIL, 2007). O Pregão Eletrônico caracteriza-se por ser inteiramente realizado via Internet, através do portal de compras do governo federal - Comprasnet ${ }^{35}$. Segundo dados do Governo Federal (BRASIL, 2007), o País economizou R\$ 1,8 bilhão com o Pregão Eletrônico em 2006. Nesse mesmo ano de 2006, o governo brasileiro adquiriu R\$ 11,1 bilhões de bens e serviços comuns através da modalidade eletrônica. Ao todo, o governo federal contratou R\$ 19,6 bilhões de bens e serviços comuns em 2006. Além da redução de custos para a administração, o Pregão Eletrônico pode agilizar e simplificar o processo de contratação, aumentar a segurança, a transparência e a democratização das compras governamentais, já que estas ocorrem pela internet.

Estudo realizado pelo Banco Mundial - BIRD (BRASIL, 2006) - indica que o sistema brasileiro de compras governamentais eletrônicas, Comprasnet, é eficiente em termos de transparência na divulgação das licitações e de seus respectivos resultados, e na utilização de métodos de licitação competitivos. Este mesmo estudo recomenda melhorias na integração do Comprasnet com os sistemas de gerenciamento de contratos e pagamentos a fornecedores, ampliando o alcance do sistema sobre a logística de compras. Ademais, sabe-se que há uma heterogeneidade na nomenclatura de produtos e serviços adotada por usuários finais e gestores do processo de compras. Esta prática gera morosidade e dificulta a integração dos sistemas de informação intra e interorganismos governamentais. Neste particular, embora o sistema Comprasnet disponha de um Catálogo de Materiais, este ainda não foi 
inteiramente incorporado pelos órgãos governamentais e é desconhecido por boa parte dos usuários finais e gestores das atividades de compras. O Catálogo de Materiais do Comprasnet adota a classificação norte-americana Federal Supply Classification ${ }^{36}$, criada e desenvolvida pelo Departamento de Defesa, com o objetivo de estabelecer e manter um sistema uniforme de identificação, codificação e catalogação para todos os órgãos componentes de sua estrutura.

O desenvolvimento de uma ontologia de produtos e serviços para a atividade de compras em geral, e do Setor Saúde em particular, poderá facilitar a prática da compra eletrônica e a integração entre os núcleos de planejamento, execução e gestão das compras inter e intra-organismos governamentais.

\section{Síntese e conclusão}

A consolidação da Tecnologia de Informação e Comunicação - TIC - foi apresentada ao longo do presente trabalho como uma tecnologia genérica (FREEMAN, 2003) que vem ocasionando expressivas transformações nas dimensões técnica, econômica, organizacional e institucional das sociedades contemporâneas. A configuração da "e-ciência" nos anos recentes confirma a vocação das atividades científicas avançadas como mercado para a TIC e mostra o potencial de transformação da TIC. A "e-ciência" exige grandes capacidades de processamento e de rede para tratar e gerenciar a enorme quantidade de dados diversos e heterogêneos gerados pelo trabalho científico, que passa a organizar-se em uma nova infraestrutura colaborativa para a produção do conhecimento científico com reflexos em outras atividades técnicas e comerciais. A evolução dessa nova organização do trabalho colaborativo demanda máquinas e redes mais potentes em arranjos do tipo Grid Computing, mas também a integração de dados e de sistemas. A construção de ontologias, as quais possuam aplicações em Sistemas Especialistas e em Web Semântica, é uma alternativa tecnológica para a interoperabilidade dos sistemas de informação e poderá favorecer essa nova organização do trabalho colaborativo em prol de uma gestão integrada de compras governamentais eletrônicas, particularmente no Setor Saúde.

\section{Notas}

1. O conceito de "paradigma tecnológico" (DOSI, 1982) equivale ao de "trajetórias naturais" (Nelson et al., 1977) e corresponde à lógica que norteia a evolução de uma determinada tecnologia.

2. Embora o conceito de "paradigma técnico e econômico" guarde semelhança com os conceitos de "paradigma tecnológico" e "trajetória tecnológica”, FREEMAN (1990) argumenta que um paradigma técnico e econômico compreende princípios orientadores que evoluem para o senso comum de engenheiros e gestores ao longo do desenvolvimento de um novo ciclo de crescimento econômico.

3. O mercado mundial da bioinformática foi estimado em U\$ 38 bilhões em 2006 (FREEMAN, 2003).
4. O termo cyberinfrastructure (NSF, 2007) é o equivalente norte-americano ao termo e-science, adotado no Reino Unido.

5. As três principais linhas de pesquisas em IA são: IA simbólica, IA conexionista e computação evolutiva. A IA conexionista trabalha com a modelagem da inteligência humana, simulando o funcionamento dos neurônios e suas interligações. A computação evolutiva é baseada em mecanismos evolutivos encontrados na natureza (BITTENCOURT, 2005).

6. O enfoque procedural manipula e raciocina sobre problemas específicos, em mundos simples, de modo a evitar os problemas de explosão combinatória, típicos dos métodos gerais (BITTENCOURT, 1998).

7. O endereço eletrônico http://www.openclinical.org/ applications.html disponibiliza informações sobre sistemas de gestão do conhecimento, sistemas de suporte à tomada de decisões e sobre aplicações em clínica médica projetadas para profissionais de saúde.

8. O Consórcio World Wide Web- W3C: http://www. w3c.org desenvolve tecnologias interoperáveis - especificações, orientação geral, software e ferramentas que favoreçam a expressão de todo o potencial da Web. O Consórcio é um fórum de informação, comércio, comunicação e compreensão mútua.

9. Recursos inclui qualquer entidade, como páginas da Web, partes de uma página da Web, dispositivos, pessoas e outros.

10. Agente(s): parte de um programa de computador executado sem o controle direto ou a supervisão do homem para alcançar metas estabelecidas por um usuário. Os agentes podem coletar, filtrar e processar informações encontradas na Web, inclusive com o auxílio de outros agentes (BERNERS-LEE, 2001).

\section{1. Mind Lab: http://www.mindswap.org}

12. Friend of a Friend: http://www.foaf-project.org/index. html.

\section{GoPubMed: http://www.gopubmed.org}

14. PubMed: http://www.ncbi.nlm.nih.gov/entrez/query. fcgi? $\mathrm{db}=$ PubMed\&itool=toolbar. PubMed é um serviço da US National Library of Medicine que inclui 16 milhões de citações do MEDLINE e outros periódicos sobre ciências da vida.

\section{GeneOntology: http://www.geneontology.org}

16. Uma lista destes produtos pode ser encontrada em http://esw.w3.org/topic/CommercialProducts\#head5ef4570c340 le6fbb8c719b398fcl692b8535d74

17. Segundo Pierre Lévy (2006), a interoperabilidade semântica equivale ao desenvolvimento da inteligência coletiva de base digital.

18. Encontramos ontologias do tipo taxionomias de categorias de páginas Web, como o site Yahoo!, e de produtos comercializados e suas características, como o site Amazon.com (NOY et al., 2001)

19. BODENREIDER et al. (2006) apresentam uma revisão do estágio atual das ontologias na biomedicina. 
20. SNOMED: http://www.snomed.org/snomedct/index. html

21. UMLS: http://www.nlm.nih.gov/pubs/factsheets/ umls.html

22. OBO: http://obo.sourceforge.net

23. OBO - ontologias genéricas específicas: http://obo. sourceforge.net/cgi-bin/table.cgi

24. GO: http://obo.sourceforge.net/cgi-bin/table.cgi

25. Plant Ontology ${ }^{\mathrm{TM}}$ Consortium: http://www.plantontology.org

26. Projeto Marine Metadata Interoperability: http://marinemetadata.org.

27. Plataforma Lattes: http://lattes.cnpq.br/index.htm

28. OPSI - http://www.opsi.gov.uk/about/index.htm

29. GovTalk: http://www.govtalk.gov.uk/howitworks/howitworks.asp

30. ESD: http://www.esd.org.uk

31. IPSV: http://www.esd.org.uk/standards/ipsv_internalvocabulary.

32. UNSPSC: http://www.unspsc.org.

33. Esta estimativa tomou por base o valor total das licitações na modalidade Pregão em 2006 (R\$ 8.833.380.000) e valor correspondente ao Ministério da Saúde no mesmo período (R\$1.945.389.000), disponíveis em http://www. comprasnet.gov.br/publicacoes/boletins/12-2006.pdf

34. Encontra-se em tramitação no Congresso Nacional, proposta de Projeto de Lei que altera dispositivos desta Lei. Por exemplo, está se propondo que qualquer modalidade de licitação estabelecida na Lei n ${ }^{\circ} 8.666$ possa ser realizada e processada por meio de sistema eletrônico integrado à Internet (BRASIL, 2007a).

35. Portal eletrônico de compras governamentais - Brasil: http://www.comprasnet.gov.br

36. http://www.dlis.dla.mil/default.asp.

\section{Referências bibliográficas}

ALVES, M.C. et al. Desenvolvimento e validação de um sistema especialista para identificar fungos na análise sanitária de sementes. Revista Brasileira de Sementes, v.28, n.1, p.176-186 (2006). Disponível em: <http://www. scielo.br/scielo.php?script $=$ sci_arttext $\&$ pid $=$ S0 101 $31222006000100025 \& \operatorname{lng}=\mathrm{pt} \& \mathrm{nrm}=\mathrm{iso}>$. Acesso em: 7 Apr. 2007.

ASSOCIAÇÃO BRASILEIRA DAS EMPRESAS DE SOFTWARE - ABES (s.d.) Entrevista com Daniel Boacnin: criatividade para crescer. s.l., s.p. Disponível em: < http://www. abes.org.br/default.aspx> . Acesso em: 8 Apr. 2007.

BAKER, M.; BUYYA, R.; LAFORENZA, D. (2002) Grids and Grid technologies for wide-area distributed computing. Software - Practice and Experience. v.32, n.15, p.141 11506, Dec. 2002. Disponível em: <http://www.gridbus. org/papers/gridtech.pdf>. Acesso em: 23 Mar. 2007.

BERNERS-LEE, T. et al. Creating a science of the Web. Science, v.313, n.5788, p.769-771 (2006). Disponível em: <http://www.sciencemag.org/cgi/content/full/313/ 5788/769 ? maxtoshow $=\&$ HITS $=10 \&$ hi... $>$. Acesso em: 18 Apr. 2007.

BERNERS-LEE, T. Web for real people. s.l.:W3C. s.p. (2005). Disponível em: <http://www.w3.org/2005/ Talks/05 1 1-keynote-tbl/> . Acesso em: 7 Apr. 2007.

BERNERS-LEE, T; HENDLER, J.; LASSILA, O. The semantic web: a new form of web content that is meaningful to computers will unleash a revolution of new possibilities. Scientific American, p.34-43, May 2001.

BITTENCOURT, G. Representação de conhecimento: da metafísica aos programas. In: SBC. (Org.). Cursos JAI'98. Belo Horizonte: UFMG. (1998) p.283-333. Disponível em: <http://www.das.ufsc.br/ gb/pg-ia/rdec. pdf>. Acesso em: 9 Mar. 2007.

BITTENCOURT, G. Computação evolutiva. In: CICLO DE CURSOS E PALESTRAS DA ENGENHARIA ELÉTRICA, 11. CIPEEL 11., 2005, Santa Catarina. UFSC: Florianópolis. p.23. Disponível em: <http://www.das.ufsc. br/ gb/CIPEEL/cevol.pdf>. Acesso em: 9 Mar. 2007.

BONDENREIDER, O.; STEVES, R. Bio-ontologies: current trends and future directions. Briefings in Bioinformatics, v.7, n.3, p.256-274, 2006.

BRASIL. Lei n. 8.666, de 21 de junho de 1993. Regulamenta o art. 37, inciso XXI, da Constituição Federal, institui normas para licitações e contratos da Administração Pública e dá outras providências. Disponível em: <http://www.planalto.gov.br/ccivil/Leis/L8666cons. htm >. Acesso em: 12 Jan. 2007.

BRASIL. Decreto n. 3.555, de 08 de agosto de 2000. Aprova o regulamento para modalidade de licitação denominada pregão, para aquisição de bens e serviços comuns. Disponível em: < https://www.planalto.gov.br/ccivil_03/ decreto/d3555.htm>. Acesso em: 12 Jan. 2007.

BRASIL. Lei n.10.520, de 17 de junho de 2002. Institui, no âmbito da união, estados, distrito federal e municípios, nos termos do art. 37, inciso xxi, da constituição federal, modalidade de licitação denominada pregão, para aquisição de bens e serviços comuns, e dá outras providências. Disponível em: <http://www.planalto.gov. br/CCIVIL/leis/2002/L10520.htm > . Acesso em: 12 Jan. 2007.

BRASIL. Decreto n. 5.450, de 31 de maio de 2005. Regulamenta o pregão, na forma eletrônica, para aquisição de bens e serviços comuns, e dá outras providências. Disponível em: <https:/www.planalto.gov.br/ccivil_03/_ Ato2004-2006/2005/Decreto/D5450.htm > . Acesso em: 12 Jan. 2007.

BRASIL. Notícias Comprasnet. 29/1 1/2006 - Estudo do banco mundial atesta eficiência do sistema comprasnet. 29 Nov. 2006. Disponível em: < http://www.comprasnet. gov.br/noticias/noticias l.asp?id_noticia $=216>$. Acesso em: 3 Apr. 2007.

BRASIL. Notícias Comprasnet. Pregão eletrônico gera economia de R\$ 1,8 bilhão em 2006. 16 Jan. 2007. Disponí- 
vel em: < http://www.comprasnet.gov.br/noticias/noticias l. asp?id_noticia $=219>$. Acesso em: 3 Apr. 2007

BRASIL. Notícias Comprasnet. Pac contempla alterações na lei geral de licitações. 23 Jan. 2007a. Disponível em: < http://www.comprasnet.gov.br/noticias/noticias 1 . asp?id_noticia $=219>$. Acesso em: 3 Apr. 2007.

CENTRO DE INFORMÁTICA - CIN/UFPE (s.d.) Engenharia de Conhecimento e Sistemas Especialistas. Recife. Disponível em: <www.di.ufpe.br/ compint/aulas-IAS/ se.ppt>. Acesso em: 11 Mar. 2007.

CHAGAS, C. S. et al. Aplicação de um sistema automatizado (ALES - Automated Land Evaluation System) na avaliação das terras das microrregiões de Chapecó e Xanxerê, Oeste Catarinense, para o cultivo de grãos. Revista Brasileira de Ciência do Solo, v.30, n.3, p.509-522, 2006. Disponível em: <http://www. scielo.br/scielo.php?script $=$ sci_arttext $\&$ pid $=$ S0 100 06832006000300>. Acesso em: 5 Apr. 2007.

COIERA, E. Clinical decision support systems. In: Guide to health informatics 2 Ed. Sydney: Arnold. (2003) Cap. 25, s.p. Disponível em: < http://www.coiera.com/ aimd.htm>. Acesso em: 4 Apr. 2007.

DOSI, G. Technological paradigms and technological trajectories. a suggested interpretation of the determinants and directions of technical change, Research Policy, v.11, n.3, p.147-208, Jun. 1982.

ENGELMORE, R.S.; FEIGENBAUM, E. Introduction. In: ENGELMORE, R.S. (Ed.) Knowledge-based systems in Japan. s.1.: WTEC Hyper-Librarian, 1993. Disponível em: <http://www.wtec.org/loyola/kb/toc. htm>. Acesso em: 30 Mar. 2007.

EUROPEAN COMMISSION. Directive 2003/98/ EC, de 17 novembro de 2003. Official Journal of the European Union, Bruxelas: EC. p.7. Disponível em: <http://europa.eu.int/eur-lex/pri/en/oj/dat/2003/1_345/ 1_34520031231 len00900096.pdf>. Acesso em: 12 Apr. 2007 .

FREEMAN, C. Policies for developing new technologies. Sussex: SPRU. 18p. SEWPS, n.98, 2003. Disponível em: < http:// www.sussex.ac.uk/Units/spru/publications/imprint/sewps/ sewp98/sewp98.pdf> . Acesso em: 6 Apr. 2007.

FREEMAN, C. Prometheus unbound. In: The economics of innovation. Aldershot: Edward Elgar Pub. Ldt. 1990. Parte V, Cap 26, p.494-507.

FURNIVAL, A.C. Delineando as limitações: sistemas especialistas e conhecimento tácito. Ciência da Informação, v.24, n.2, s.p. 1995. Disponível em: <http://www. scielo.br/scielo.php?script $=$ sci_arttext $\&$ pid $=$ S0 100 $73862000000400003 \& \operatorname{lng}=\mathrm{en}_{\mathrm{N}} \mathrm{nrm}=\mathrm{iso}>$. Acesso em: 9 Mar. 2007.

GIBOSHI, M.L.; RODRIGUES, L.H.A.; LOMBARDI NETO, F. Sistema de suporte à decisão para recomendação de uso e manejo da terra. Revista Brasileira de Engenharia Agrícola e Ambiental, v.10, n.4, p.861866, 2006. Disponível em: < http://www.scielo.br/scielo. php? script $=$ sci_arttext $\&$ pid $=$ S 1 41 5-66200600040001 2\&lng=pt\&nrm=iso > . Acesso em: 7 Apr. 2007.

GLOBE, C. Sense and Sensibility: Semantic Infrastructure for Bioinformatics. In: INITIATIVE FOR INNOVATIVE COMPUTING. Harvard. 31 Jan. 2007. Disponível em: <http://www.cs.man.ac.uk/ carole/talks/index.html >. Acesso em: 7 Abr. 2007.

GROSOF, B.N. et al. Description logic programs: Combining logic programs with description logic. In: PROC. OF THE TWELFTH INTERNATIONAL CONFERENCE ON WORLD WIDE WEB, Budapest: ACM, 20-24 May. p.48-57, 2003.

GRUBER. T.R. A translation approach to portable ontologies. Knowledge Acquisition, v.5, n.2, p.199-220, 1993.

HENNEMANN, F.A. et al. Um sistema híbrido de apoio à decisão formado por redes de Petri, simulação e sistema especialista. Revista Controle \& Automação, v.17, n.1, p.10-23, 2006. Disponível em: <http://www. scielo.br/scielo.php?script $=$ sci_arttext $\&$ pid $=$ S0103$17592006000100002 \& \operatorname{lng}=\mathrm{en} \& n r m=$ iso $>$. Acesso em: 7 Apr. 2007.

HERMAN, I. Introduction to the Semantic Web: tutorial on Semantic Web technologies. In: INTERNATIONAL CONFERENCE ON SEMANTIC WEB \& DIGITAL LIBRARIES. Bangalore. 21 Fev. 2007. Disponível em: <http://www.w3.org/2007/Talks/022 1-Bangalore-IH/>. Acesso em: 9 Apr. 2007.

HEY, T.; TREFETHEN, A.E. The UK e-science core programme and the Grid. Future Generation Computing Systems, v.18, p.1017-34, 2002. Disponível em: < http://www.rcuk.ac.uk/cmsweb/downloads/rcuk/research/esci/coreproggrid.pdf>. Acesso em: 29 Mar. 2007.

HINE, C.M. Preface. In: New infrastructures for knowledge production; understanding e-science. London: INFOSCL. 2006, p.vii-xvii.

LÉVY, P. IEML: computational semantics in the service of collective intelligence. Otawa: CRC/FRSC. p. 9, 2006 (translated by Michele Healy). Disponível em: $<$ http://www.ieml.org/IMG/pdf/vision-ieml-Initiativeen.pdf>. Acesso em: 8 Apr. 2007.

LINDSAY, R.K. DENDRAL: a case study of the first expert system for scientific hypothesis formation. Artificial Intelligence v.61, p.209-261, 1993. Disponível em: < http://profiles.nlm.nih.gov/BB/A/B/O/M/_bbabom. pdf $>$. Acesso em: 8 Apr. 2007.

LUGER, G.F. Artificial intelligence: its roots and scope. In: Artificial intelligence: structures and strategies for complex problem solving. 5 ed. s.l.: Addison-Wesley. 2005. p.1-34. Disponível em: <http://www.cs.unm. edu/ luger/ai-final/chapterl.html $>$. Acesso em: 30 Mar. 2007.

MACKWORTH, A.K. AI Overview. AAAI, 2007. Disponível em: <http://www.aaai.org/AITopics/html/overview. html>. Acesso em: 24 Mar. 2007. 
MAZIERO, N. L. et al. A feature-based object-oriented expert system to model and support product design. Journal of the Brazilian Society of Mechanical Sciences, v.22, n.4. s.p., 2000. Disponível em: <http://www. scielo.br/scielo.php?script $=$ sci_arttext \& pid $=$ S0 100 $73862000000400003 \& \operatorname{lng}=\mathrm{en} \& \mathrm{nrm}=\mathrm{iso}>$. Acesso em: 7 Abr. 2007.

MCCARTHY, J.; HAYES, P.J. (1969) Some philosophical problems from the standpoint of artificial intelligence. In: MICHIE, D.; MELTZER, B. (Eds.) Machine intelligence. Edinburgh: Edinburgh University Press. p.463-502. Disponível em: <http://www-formal.stanford.edu/jmc/mcchay69/mcchay69.html>. Acesso em: 24 Mar. 2007.

MCCARTHY, John. (2004) What is artificial intelligence? Standford: Computer Science Department Stanford University. Disponível em: <http://www-formal.stanford. edu/jmc/whatisai.html>. Acesso em: 24 Mar. 2007.

MENDES, Raquel Dias. (1997) Inteligência artificial: sistemas especialistas no gerenciamento da informação. Ciência da Informação, v.26, n.1, p.39-45, Jan.-Abr. 1997. Disponível em: <http://www.scielo.br/scielo. php? script $=$ sci_arttext $\&$ pid $=$ S0100-1965199700010 0006\&lng=en\&nrm=iso $>$. Acesso em: 7 Apr. 2007.

NELSON, R.R.; WINTER, S.G. (1977) In search of useful theory of innovation, Research Policy, v.6, n.1, p.36-76, Jan. 1977.

NOY, N.F; McGUINNESS, D.L. (2001) Ontology Development 101: A Guide to Creating Your First Ontology. S. Stanford University. p.25. Disponível em: <http://www.ksl.stanford.edu/people/dlm/papers/ontology 101/ontology101-noy-mcguinness.html>. Acesso em: 9 Mar. 2007.

NSF (2007) Cyberinfrastructure vision for 21 st century discovery. Arlington. 64 p. Disponível em: <http://www.nsf.gov/pubs/2007/nsf0728/nsf0728.pdf>. Acesso em: 8 Apr. 2007.

PACHECO, Roberto Carlos dos Santos; KERN, Vinícius Medina (2001) Transparência e gestão do conhecimento por meio de um banco de teses e dissertações: a experiência do PPGEP/UFSC. Ciência da Informação, v.30, n.3, p.64-72, Sep.-Dec. 2001. Disponível em: <http://www. scielo.br/scielo.php? script $=$ sci_arttext $\&$ pid $=$ S0 100 $19652001000300009 \& \operatorname{lng}=\mathrm{pt} \& n \mathrm{~mm}=\mathrm{iso}>$. Acesso em: 6 Mar. 2007.

PEREZ, C. (1986) Las nuevas tecnologías; una visión de conjunto. In: OMINAMI, C. (Ed). La tercera revolución industria; impactos internacionales del actual viraje tecnológico. Buenos Aires: Grupo Editor Latinoamericano. cap. 2 , p. $43-89$

RAZZOUK, D. et al. (2006) Decision support system for the diagnosis of schizophrenia disorders. Brazilian Journal of Medical and Biological Research, v.39, n.1, p.119-128. Disponível em: < http://www. scielo.br/scielo.php? script $=$ sci_arttext $\&$ pid $=$ S0 100 $879 X 2006000100014 \& \operatorname{lng}=\mathrm{en} \& n r m=i s o>$. Acesso em: 7 Apr. 2007.

REDFEARN, J. (s.d.) Highlights from de UK e-science programme. The Research Councils UK: s.l. 4p. Disponível em: <http://www.rcuk.ac.uk/cmsweb/downloads/rcuk/research/esci/escihighlights.pdf $>$. Acesso em: 23 Mar. 2007.

STUDER, R.; BENJAMINS, R.; FENSEL, D. Knowledge engineering principles and methods. Data and Knowledge Engineering (DKE), v.25, n.1/2, p.161$197,1998$.

WELTY, C. Ontology Research. Al Magazine, v.24, n.3, p.1-12, 2003. Disponível em: <http://www.aaai. org/AlTopics/assests/PDF/AIMag24-03-002.pdf>. Acesso em: 9 Apr. 2007. 


\section{Sobre os autores}

\section{Laura Viana}

Doutora em Engenharia de Produção pela Coordenação dos Programas de Pós-Graduação em Engenharia (COPPE) da Universidade Federal do Rio de Janeiro. Atualmente é analista em Ciência e Tecnologia da Fundação Oswaldo Cruz. Tem experiência profissional nos setores público e privado, com ênfase em inovação tecnológica e organização industrial aplicada às indústrias de tecnologia da informação e comunicação, farmacêutica e de alimentos, em estudos e projetos sobre inovação em serviços, pesquisa e desenvolvimento tecnológico e políticas públicas.

\section{Olga Nabuco}

Doutora em Engenharia Mecânica pela Universidade Estadual de Campinas e pesquisadora do Centro de Pesquisa Renato Archer. Atualmente é pesquisadora visitante do Laboratoire D'Analyse Et D'Architecture Des Systemes - Centre National de la Recherche Scientifique (LAAS/CNRS). Sua área de atuação caracteriza-se, principalmente, pelos estudos de modelos de representação de conhecimento, ontologias, compartilhamento de conhecimento, arquiteturas orientadas a serviço, modelos de negócios e web semântica. 